\title{
Volatile organic compounds profile during milk fermentation by Lactobacillus pentosus and correlations between volatiles flavor and carbohydrate metabolism
}

\author{
D. D. Pan, ${ }^{\star} \dagger^{1}$ Z. Wu, $\dagger$ T. Peng, $\dagger$ X. Q. Zeng, ${ }^{*}$ and H. Li† \\ ${ }^{*}$ Food Science and Technology Department of the Marine Science School, Ningbo University, Ningbo 315211, P. R. China \\ †Food Science and Nutrition Department, Nanjing Normal University, Nanjing 210097, P. R. China
}

\begin{abstract}
Flavor, as one of the most important properties determining the acceptability and preference of fermented milks, is influenced by compositional and processing factors. In this study, we focused on the volatile organic compounds related to flavor during milk fermentation by Lactobacillus pentosus according to electronic nose analysis. Xylose ( $1 \%$ addition) metabolized by $L b$. pentosus strongly affects the flavor of yogurt, with the potent volatile organic compounds of ethanol $(3.08 \%)$, 2,3-butanedione $(7.77 \%)$, and acetic acid $(22.70 \%)$ detected using solid-phase microextraction coupled with gas chromatography-mass spectrometry analysis. Sensory analysis also showed skimmed yogurt fermented by $L b$. pentosus with $1 \%$ xylose had the unique scores of sourness (acetic acid) and butter flavor (2,3-butanedione). Furthermore, $\alpha$-acetolactate synthase and $\alpha$-acetolactate decarboxylase in carbohydrate metabolism play important roles in milk fermentation. Under

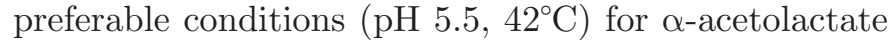
synthase and $\alpha$-acetolactate decarboxylase, the relative content of potent flavor compound 2,3-butanedione was $10.13 \%$, which was $2.55 \%$ higher than common culture condition $\left(\mathrm{pH} 4.5,37^{\circ} \mathrm{C}\right)$, revealing that xylose metabolized by $L b$. pentosus has potential values for the milk product industry, such as the acceptability and preference of fermented milk product.
\end{abstract}

Key words: Lactobacillus pentosus, volatile organic component, electronic nose, solid-phase microextraction coupled with gas chromatography-mass spectrometry (SPME-GC-MS)

\section{INTRODUCTION}

Cultured dairy products are widely consumed due to health and nutrition claims and also for their sensory properties. Naturalness and agreeable taste make

Received June 12, 2013.

Accepted October 29, 2013.

${ }^{1}$ Corresponding author: daodongpan@163.com yogurt an attractive food for consumption. The primary sensory attributes of yogurt include texture, taste, aroma, and flavor (Salvador and Fiszman, 2004; Sodini et al., 2006). Flavor, as one of the most important properties determining the acceptability and preference of fermented milks, is influenced by compositional and processing factors (Soukoulis et al., 2007). Milk fermentation is a complex process for the transformation of milk into yogurt. Compositional factors, namely nonvolatile acids (lactic and pyruvic), volatile acids (butyric and acetic), carbonyl compounds (acetaldehyde and diacetyl), and miscellaneous compounds, are always influenced by pasteurization, fermentation, and storage (Hugenholtz et al., 2000). Considering that the native volatile constituents in milk may change during the yogurt fermentation period, the chemical ingredients of milk base, type of milk, processing methods, and types of starter culture need to be carefully investigated for high-quality yogurt production (Kühn et al., 2006). Meanwhile, with the increasing demand for low-fat food products, more attention should be paid to yogurt with improved compositional and nutritional properties (Abete et al., 2011).

Currently, starters containing mainly Lactobacillus delbrueckii ssp. bulgaricus and Streptococcus thermophilus are used widely in the industrial production of yogurt fermentation and quality improvement (HerveJimenez et al., 2009). The difference in aroma has been attributed to the presence of different bacteria. The basic volatile organic compounds (VOC) participating in the formation of the flavor of typical Bulgarian yogurt are carbonyl compounds, such as acetaldehyde, acetone, 2-butanone, diacetyl, ethyl acetate, and ethanol (Cheng, 2010). Low-molecular VOC in milk supplemented with the strain Bifidobacterium animalis ssp. lactis $\mathrm{Bb}-12$ with or without fermentation were also reported by Zareba et al. (2012). Yogurt flavor is the critical factor in both product evaluation and consumer acceptability. The correlations between flavor properties and VOC provide the basis for making qualified decisions in producing high-quality yogurt. The evolution of VOC during fermentation can provide useful 
information about the quality of the final product and the possible inefficiencies in the process of incubation (Soukoulis et al., 2010).

Milk is an extremely complicated entity comprising lipids, proteins, carbohydrates, and minerals. Over 400 volatile compounds have been identified in lactic acid bacteria (LAB) fermented milk products. Lactic acid bacteria have the capacity of adopting microbial, enzymatic, or chemical transformation to degrade lactose, lipids, citric acid, and proteins/amino acids in milk (Law and Haandrikman, 1997). During fermentation, the differences between the VOC of yogurt and milk are most likely generated by the metabolism of LAB. The carbon-hydrogen transport systems in LAB are specific for carbohydrate transportation and are adenosine triphosphate dependent and they are able to activate complex enzymatic systems in the milk fermentation process (Rabot et al., 2010).

When measuring the relative concentrations of a large number of metabolic enzymes in the processing of fermented milk, it is essential to come to a comprehensive understanding of evolution of VOC during fermentation. $\alpha$-Acetolactate synthase (ALS) and $\alpha$-acetolactate decarboxylase (ALDB) are 2 essential enzymes when pyruvate is channeled to acetoin or diacetyl (Lee et al., 2013). The progress in identifying the key flavor-related compounds and their origins will enable manufacturers to produce more uniform dairy products.

Lactose, a $\beta$-1,4-linked disaccharide of $\beta$-D-galactose and $\alpha / \beta$-D-glucose, is a common constitution in dairy product. Xylose is also abundant in nature and supposedly has several health-promoting effects, such as being low caloric, low glycemic, low insulinemic, antimicrobial, and prebiotic. These effects could occur in milk fermentation by Lactobacillus pentosus (Chaillou et al., 1999). However, little attention has been paid to the VOC changes during milk fermented by $L b$. pentosus and related metabolic pathways. The use of traditional starters limits the organoleptic variation of the end products (Ao et al., 2012). Recently, we characterized a strain of $L b$. pentosus, which could produce specific flavor in fermented milk. For the acceptability and preference of fermented milks, we investigated VOC differences among various kinds of milk products fermented by $L b$. pentosus using principal components analysis (PCA) according to an electronic nose (Enose) analysis and determination of VOC by solidphase microextraction coupled with GC-MS (SPMEGC-MS). Furthermore, the fermentation conditions that affect the activity of ALS and ALDB were also explored. This research will contribute to the selection and application of this new starter in the traditional milk fermentation process.

\section{MATERIALS AND METHODS}

\section{Strain and Medium}

The strain was isolated from traditional fermented milk products in Xinjiang Province (China) and classified as $L b$. pentosus (ATCC8041) by $16 \mathrm{~S}$ rRNA gene sequence analysis (Peng et al., 2011). Lactobacillus pentosus was cultured at $37^{\circ} \mathrm{C}$ in de Man, Rogosa, and Sharpe (MRS) medium and stored at $-80^{\circ} \mathrm{C}$ in culture plus glycerol $(20 \%, \mathrm{vol} / \mathrm{vol})$ for further use.

\section{Reagents}

Milk powder and yogurt (fermented with Lactobacillus bulgaricus and Strep. thermophilus) were purchased from Bright Dairy Co. (Shanghai, China). Thiamine pyrophosphate, flavin adenine dinucleotide, oxaloacetate, and Coomassie brilliant blue G250 were purchased from Sigma-Aldrich Co. (Shanghai, China); BSA was purchased from Sangon Biotech (Shanghai, China). Other reagents were all analytical grade.

\section{Yogurt Manufacture}

Bovine milk, including cream milk (CM) and skim milk (SM; $1.5 \%$ milk fat), was heated for 30 min using batch pasteurization $\left(75^{\circ} \mathrm{C}\right)$ and cooled at $37^{\circ} \mathrm{C}$ in a water bath. Each milk sample was divided into 3 groups ( $150 \mathrm{~mL}$ per flask), and inoculated with $L b$. pentosus $(2 \%, \mathrm{vol} / \mathrm{vol})$ at $37^{\circ} \mathrm{C}$ for $12 \mathrm{~h}$; when the $\mathrm{pH}$ reached 4.5 , the cured-milk samples were stored at $4^{\circ} \mathrm{C}$ for further analysis. For different samples, 1\% lactose or $1 \%$ xylose was added to the milk samples during milk fermentation.

\section{E-Nose Analysis of Milk Samples}

Samples were collected from CM, SM, cream yogurt (CY), skimmed yogurt (SY), skimmed yogurt fermented by $L b$. pentosus with $1 \%$ xylose (SYX), and plain yogurt from the market fermented by Lb. bulgaricus and Strep. thermophilus (PY). Linear discriminant analysis (LDA) and PCA of main VOC from various fermented milk products were performed with an Airsense PEN 3 E-nose (Airsense Analytics GmbH, Schwerin, Germany). Each sample $(50 \mathrm{~mL})$ was placed in $125-\mathrm{mL}$ Pyrex 4980 flasks with silicone caps and then introduced to the sampling apparatus of the E-nose ( $\mathrm{Yu}$ and Wang, 2007; Mamat et al., 2011). The E-nose response to milk samples at different temperatures, including 20, 40,60, and $80^{\circ} \mathrm{C}$, were analyzed according to the LDA.

\section{SPME-GC-MS Analysis of VOC}

Volatile organic compound identification was performed by SPME-GC-MS. Carboxen/polydimethylsi- 


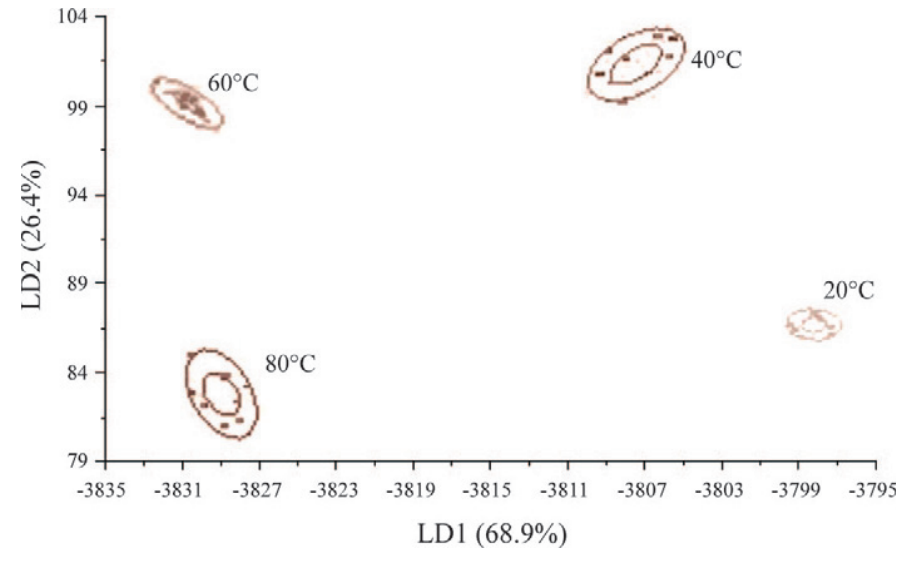

Figure 1. Linear discriminant (LD) analysis score plot of the electronic nose response to milk samples at different temperatures. Color version available in the online $\mathrm{PDF}$.

loxane (CAR/PDMS, $75 \mu \mathrm{m})$ was selected and mounted in an SPME manual holder (Supelco Inc., Bellefonte, PA), based on the broad retention over a large range of polarity and sensitive to both polar and nonpolar aliphatic aldehydes, according to the research of Roberts et al. (2000).

The optimal parameters for VOC analysis were as follows: the sample $(5 \mathrm{~g})$ was placed in a 25 -mL vial sealed with a polytetrafluoroethylene septum and bathed at $55^{\circ} \mathrm{C}$ for $10 \mathrm{~min}$ to equilibrate. The septum was then pierced with the SPME needle and the fiber was exposed to the sample headspace $(10 \mathrm{~mL})$ for 45 min. After extraction, the fiber was inserted into the injection port of the gas chromatograph immediately and desorbed at $250^{\circ} \mathrm{C}$ for $5 \mathrm{~min}$. The GC-MS analysis was

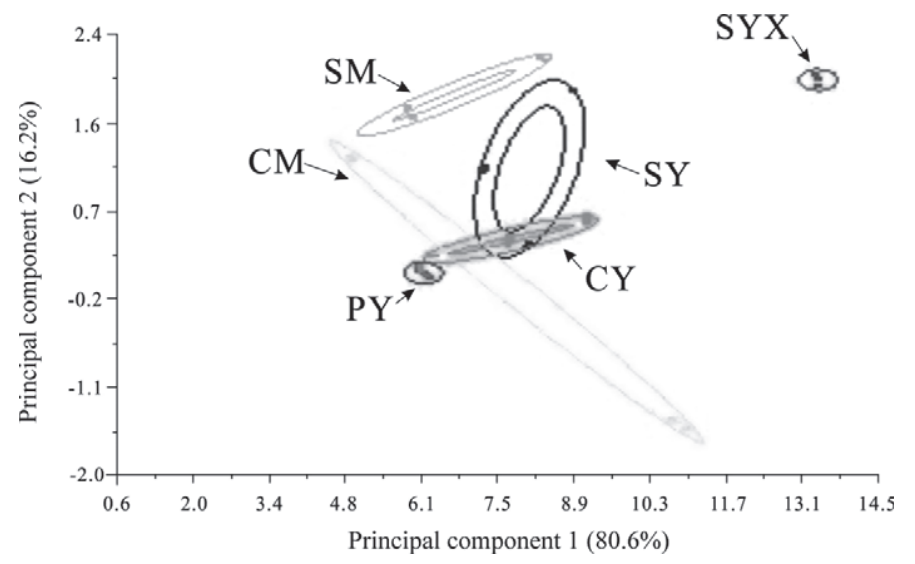

Figure 2. Principal components analysis score plot of the electronic nose response to different milk samples. $\mathrm{SM}=$ skim milk; $\mathrm{CM}=$ cream milk; PY = plain yogurt from market fermented by Lactobacillus bulgaricus and Streptococcus thermophilus; $\mathrm{CY}=$ cream yogurt; $\mathrm{SY}=$ skimmed yogurt; SYX = skimmed yogurt fermented by Lactobacillus pentosus with $1 \%$ xylose.
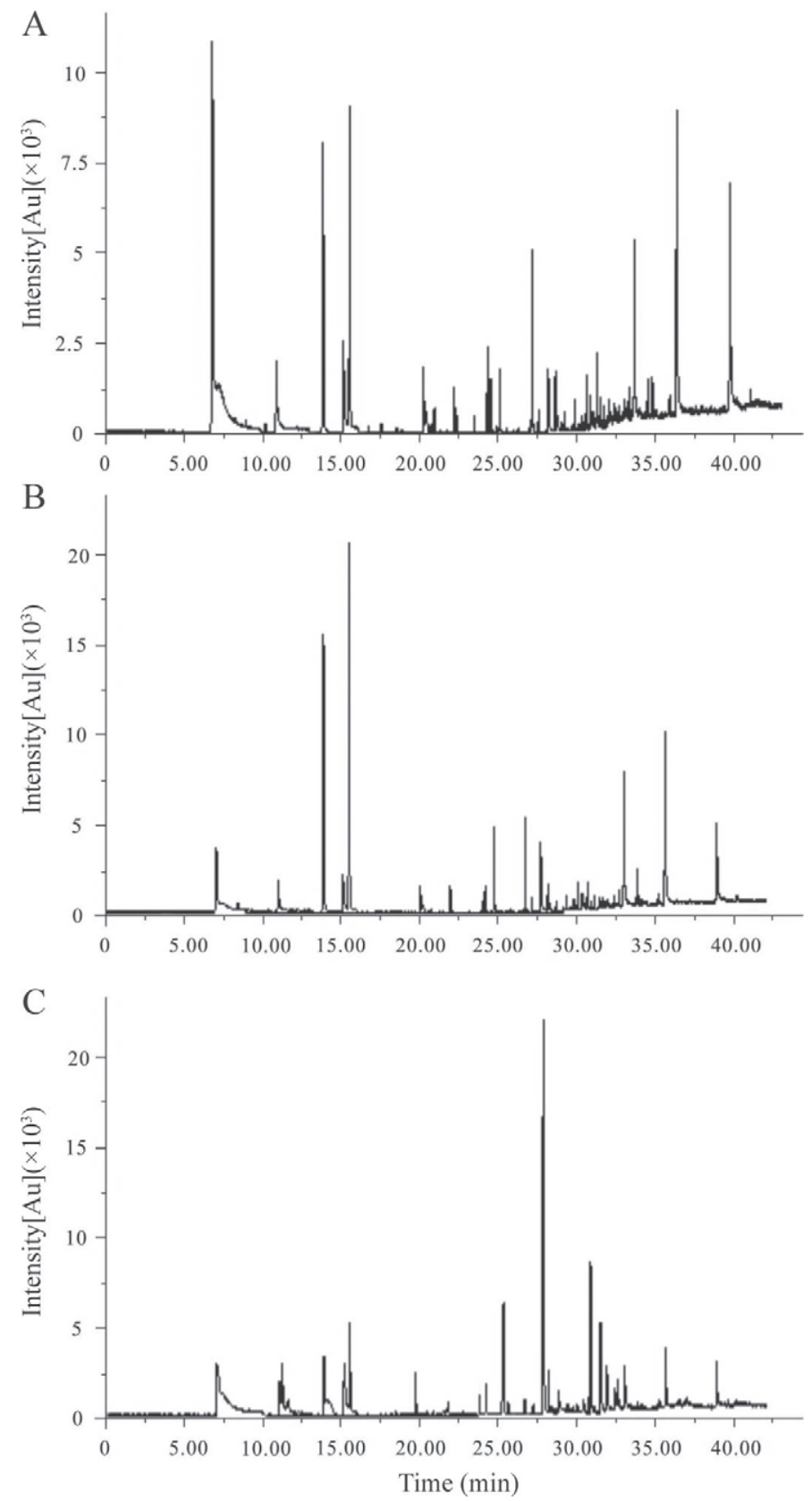

Figure 3. Solid-phase microextraction coupled with GC-MS (SPME-GC-MS) chromatograms of volatile flavor compounds of different yogurt products. (A) Skimmed yogurt fermented by Lactobacillus pentosus; (B) skimmed yogurt fermented by $L b$. pentosus with $1 \%$ lactose; (C) skimmed yogurt fermented by $L b$. pentosus with $1 \%$ xylose. $\mathrm{Au}=$ arbitrary units.

performed as described by Majcher and Jeleń (2011), with some modifications. The GC oven temperature program was held at $40^{\circ} \mathrm{C}$ for $4 \mathrm{~min}$ and increased from 40 to $60^{\circ} \mathrm{C}$ at a rate of $5^{\circ} \mathrm{C} / \mathrm{min}, 60$ to $120^{\circ} \mathrm{C}\left(6^{\circ} \mathrm{C} /\right.$ $\min )$, and 120 to $230^{\circ} \mathrm{C}\left(10^{\circ} \mathrm{C} / \mathrm{min}\right)$. The GC-MS transfer line was maintained at $250^{\circ} \mathrm{C}$. Helium flow rate was $0.8 \mathrm{~mL} / \mathrm{min}$. Mass spectra were collected in electron 
Table 1. Volatile organic compounds of yogurt samples ${ }^{1}$

\begin{tabular}{|c|c|c|c|c|}
\hline \multirow[b]{2}{*}{ Volatile organic compound } & \multirow[b]{2}{*}{$\begin{array}{l}\text { Retention } \\
\text { time (min) }\end{array}$} & \multicolumn{3}{|c|}{ Relative content (\%) } \\
\hline & & $\begin{array}{c}\text { Lactobacillus } \\
\text { pentosus }\end{array}$ & $\begin{array}{l}\text { Lb. pentosus } \\
+ \text { lactose }^{3}\end{array}$ & $\begin{array}{l}\text { Lb. pentosus } \\
+\mathrm{xylose}^{4}\end{array}$ \\
\hline Acetaldehyde & 8.35 & $\mathrm{ND}^{5}$ & 0.88 & ND \\
\hline Cyclotrisiloxane, hexamethyl- & 9.84 & 3.13 & 2.3 & 9.06 \\
\hline Acetone & 10.25 & ND & 0.23 & ND \\
\hline Ethanol & 13.74 & 11.00 & 18.09 & 3.08 \\
\hline Cyclotetrasiloxane, octamethyl- & 14.98 & 3.35 & 2.82 & 4.08 \\
\hline Butanoic acid, methyl ester & 15.35 & 9.86 & 18.34 & ND \\
\hline Cyclopentasiloxane, decamethyl- & 19.15 & 2.94 & 1.25 & 2.44 \\
\hline$o$-Xylene & 19.38 & 0.69 & ND & 0.88 \\
\hline 2-Heptanone & 20.71 & 1.15 & 1.04 & ND \\
\hline Styrene & 22.62 & 2.15 & 0.44 & ND \\
\hline Cyclohexasiloxane, dodecamethyl- & 23.18 & 1.27 & 0.95 & 1.19 \\
\hline 2,3-Butanedione & 24.91 & 1.74 & 3.75 & 7.77 \\
\hline 2-Nonanone & 25.76 & 3.67 & 3.01 & 0.61 \\
\hline Acetic acid & 27.17 & 2.54 & 4.25 & 22.70 \\
\hline 2-Undecanone & 29.27 & 1.01 & 0.98 & ND \\
\hline Butanoic acid & 29.81 & ND & 0.96 & ND \\
\hline 2-Furanmethanol & 30.57 & ND & ND & 6.44 \\
\hline Oxime-, methoxy-phenyl- & 31.20 & 1.05 & ND & 5.66 \\
\hline 2-Tridecanone & 31.95 & 0.74 & 0.61 & $\mathrm{ND}$ \\
\hline Octanoic acid & 32.39 & 9.61 & 16.57 & 5.23 \\
\hline Hexanoic acid & 32.69 & 4.74 & ND & ND \\
\hline Butylated hydroxytoluene & 33.15 & 0.71 & 1.19 & ND \\
\hline$n$-Decanoic acid & 38.11 & 8.90 & 5.40 & 2.75 \\
\hline
\end{tabular}

${ }^{1}$ Values in bold for ethanol, 2,3-butanedione, and acetic acid indicate that their relative contents showed differences $(P<0.05)$ during carbohydrate metabolism among the 3 samples..

${ }^{2}$ Skimmed yogurt fermented by $L b$. pentosus.

${ }^{3}$ Skimmed yogurt fermented by $L b$. pentosus with $1 \%$ lactose.

${ }^{4}$ Skimmed yogurt fermented by $L b$. pentosus with $1 \%$ xylose.

${ }^{5} \mathrm{ND}=$ not detected.

ionization mode, with an iron source temperature of $200^{\circ} \mathrm{C}$. The mass range was set at 33 to $450 \mathrm{~m} / z$, with $3.50 \mathrm{scans} / \mathrm{s}$. All compounds were semi-quantified (3 replicates) and the results are presented as retention time and relative peak area.

\section{Sensory Evaluation}

A sensory analysis was used to evaluate differences among the 3 fermented milk products according to the method of Chen et al. (2011). Ten trained panelists were invited and asked to rate samples for firmness, sweetness, sourness, astringent, and butter flavor using a 10-point intensity scale anchored with the words "low" (1-3), "medium" (5), and "high" (8-10) for each fermented milk product. Sensory evaluation was conducted in individual booths to prevent rate score bias. Scores are presented as mean \pm standard error. Spring water (Nongfu Spring, Zhejiang, China) was provided for cleaning the palate between samples.

\section{ALS and ALDB Assays}

Considering the important role of ALS and ALDB in carbohydrate metabolism, especially in milk fermen- tation by LAB, the activities of ALS and ALDB in fermentation processing were measured in this study. Cells $(2 \mathrm{~g})$ were resuspended in $20 \mathrm{~mL}$ of potassium phosphate buffer ( $\mathrm{pH} 7.2)$ and incubated with lysozyme (glycoside hydrolase) at $37^{\circ} \mathrm{C}$ for $2 \mathrm{~h}$. Suspensions were cooled, centrifuged at $12,000 \times g$ for 5 min at $4^{\circ} \mathrm{C}$, and the supernatant was collected to determine the in vivo enzyme concentration. The concentration of ALS and ALDB was determined using the Bradford method, with BSA as the standard. The activity of ALS and ALDB were assayed as described previously (Hugenholtz and Starrenburg 1992; Monnet et al., 1994). One unit of ALS (ALDB) activity represented the formation of $1 \mu \mathrm{mol}$ of diacetyl per minute.

\section{RESULTS AND DISCUSSION}

\section{Milk Sample Analysis With the E-Nose}

The E-nose is a powerful tool for the monitoring of VOC formed during milk fermentation. The LDA for the E-nose measurement shows that samples at different temperatures could be identified significantly from the within-class distance and between-class distance (Figure 1). Results of LDA scores of the E-nose response 


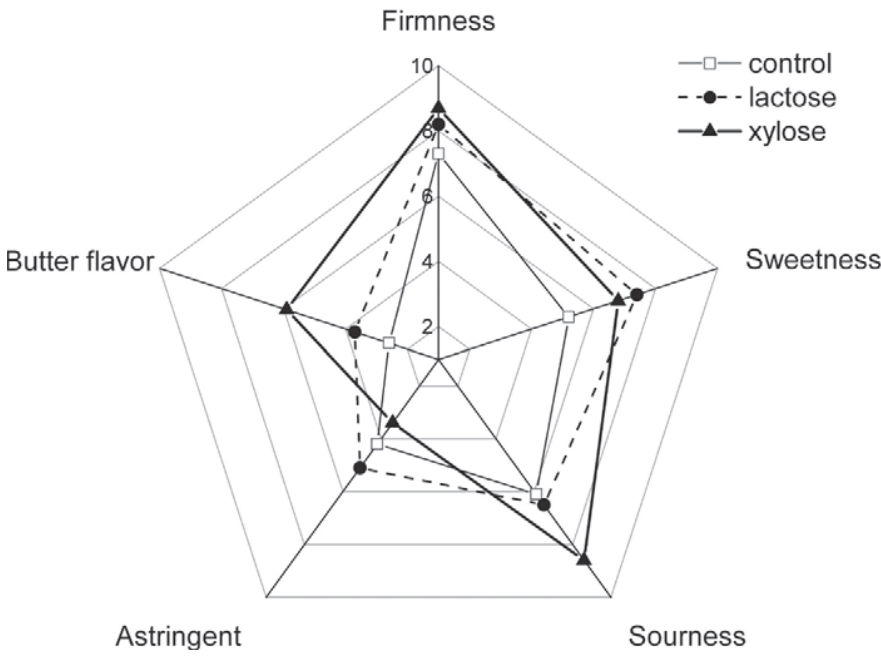

Figure 4. Radar plot of sensory scores of 3 different fermented milk samples. Control = skimmed yogurt fermented by Lactobacillus pentosus; lactose $=$ skimmed yogurt fermented by $L b$. pentosus with $1 \%$ lactose; xylose $=$ skimmed yogurt fermented by $L b$. pentosus with $1 \%$ xylose.

to milk sample in the 4 temperature groups $(20,40,60$, and $80^{\circ} \mathrm{C}$ ) are classified clearly in the plot. The withinclass distance at $20^{\circ} \mathrm{C}$ was the shortest among the 4 detected temperatures, followed by $60^{\circ} \mathrm{C}$. To minimize the within-class distance and maximize the between-class distance, we chose $60^{\circ} \mathrm{C}$ for the temperature parameter in the following E-nose analysis. The analytical results for the response value at $60^{\circ} \mathrm{C}$ were better than those at other collection temperatures (Grigioni et al., 2000).

Principal components analysis was performed to give an overall picture of the VOC distribution among the nonfermented and fermented milk samples (Figure 2 ). From the within-class distance and between-class distance among different samples it is possible to distinguish the different characteristics among various samples (Delgado et al., 2011). The first principal component (PC) explained $80.64 \%$ of the variation across the samples, whereas the PC2 explained $16.21 \%$ of the variance in different samples. Principal component 1 and $\mathrm{PC} 2$ accounted together for almost the $96.85 \%$ of the total data variance. Plots of SYG were more convergent and are clearly separated from samples CM, SM, CY, SY, and PY, whereas some of the clusters, such as CY, SY, and CM, appeared to be closer than other samples. The sensory properties of dairy products depend largely on the relative balance of VOC derived from carbohydrates in the milk (Cheng, 2010). For the SYX sample, fermented by $L b$. pentosus with $1 \%$ xylose, the flavor was unique among all the detected samples. Lactobacillus pentosus is capable of generating energy from homo- to heterofermentative degradation in the absence of glucose (De Vuyst and Vancanneyt,

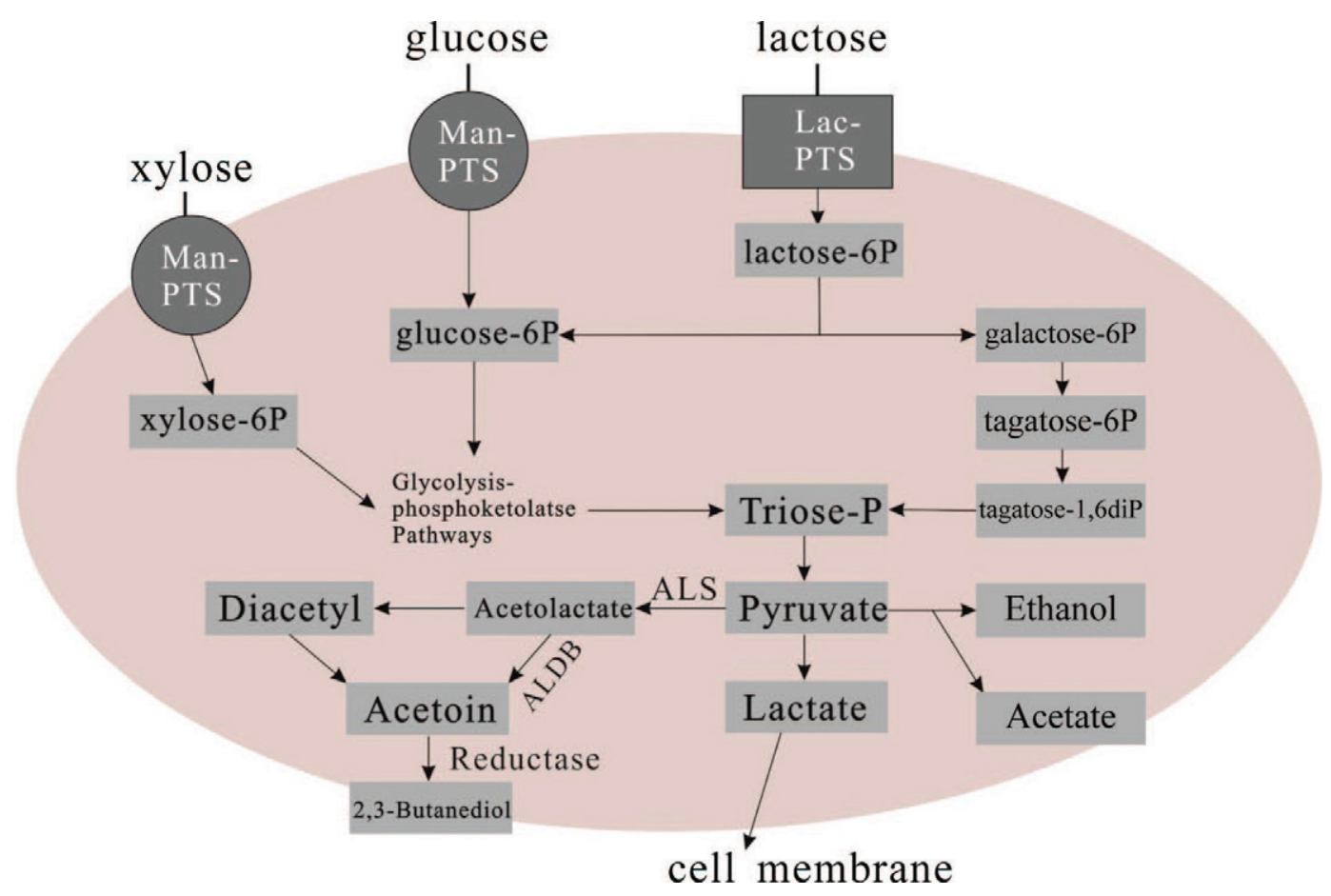

Figure 5. Glucose, lactose, and xylose involved in carbohydrate metabolism during milk fermentation by Lactobacillus pentosus. Man-PTS $=$ mannose phosphotransferase system; Lac-PTS = lactose phosphotransferase system; P = phosphate; ALS = $\alpha$-acetolactate synthase; ALDB $=\alpha$-acetolactate decarboxylase; $\operatorname{diP}=$ diphosphate. Color version available in the online PDF. 
2007). According to the PCA among different samples, xylose metabolized by $L b$. pentosus strongly affected the flavor of yogurt compared with other fermented milk products.

\section{VOC Analysis}

Volatile organic compounds of fermented milk are quite complex; only a few compounds have a major effect on flavor development. In the present study, SPME-GC-MS was used to identify the VOC present in the milk fermentation and to detect their diverse distribution in different yogurt samples. A profound result of essential VOC profiles and their relative contents (RC) are summarized in Figure 3 and Table 1. Twentyfour VOC detected in 3 samples were divided into 7 categories: 6 acids, 6 ketones, 4 cyclohexasiloxanes, 3 benzenes, 3 alcohols, 1 aldehyde, and 1 miscellaneous
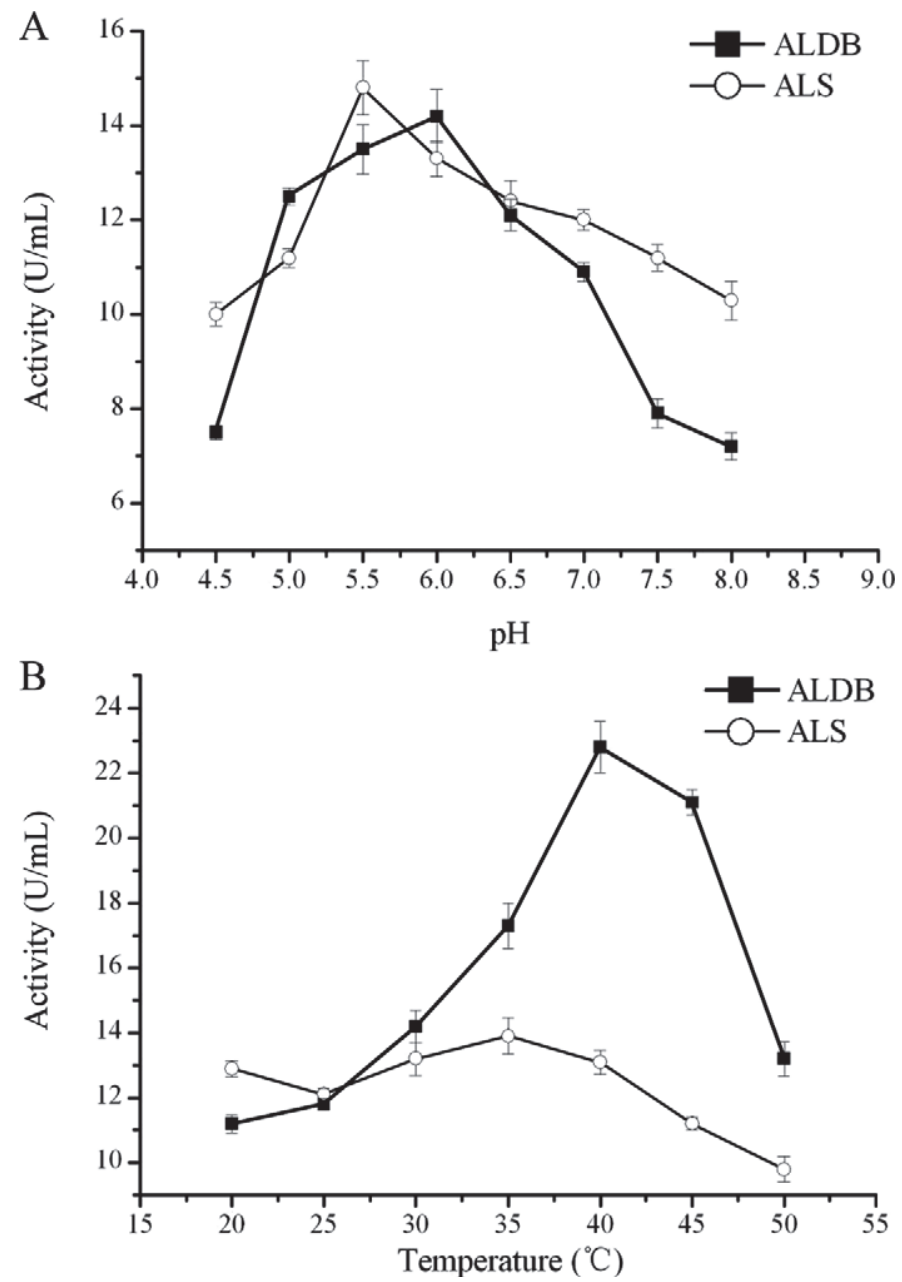

Figure 6. $\alpha$-Acetolactate synthase (ALS) and $\alpha$-acetolactate decarboxylase (ALDB) assayed in vitro as a function of $\mathrm{pH}$ and temperature. Error bars represent SD.
Table 2. Potent flavor compounds of a yogurt sample ${ }^{1}$ identified in solid-phase microextraction coupled with GC-MS (SPME-GC-MS)

\begin{tabular}{lcc}
\hline $\begin{array}{l}\text { Volatile } \\
\text { component }\end{array}$ & $\begin{array}{c}\text { Retention } \\
\text { time (min) }\end{array}$ & $\begin{array}{c}\text { Relative } \\
\text { content }(\%)\end{array}$ \\
\hline 2,3-Butanedione & 24.91 & 10.13 \\
Acetic acid & 27.17 & 15.17 \\
\hline
\end{tabular}

${ }^{1}$ Skimmed yogurt fermented by Lactobacillus pentosus with $1 \%$ xylose.

compound. The RC of potent flavor compounds ethanol, 2,3-butanedione, and acetic acid showed significant differences during carbohydrate metabolism among the 3 samples. Previous research found that the main subproduct fermented by $L b$. pentosus was acetic acid (Bustos et al., 2005), which was consistent with the results revealed in our study; the $\mathrm{RC}$ of acetic acid in skimmed yogurt fermented by $L b$. pentosus with $1 \%$ xylose was $22.70 \%$, which was higher than the other 2 samples. Meanwhile, a small lactose concentration was detected in the milk fermentation (data not shown), which gave some evidence of $L b$. pentosus having lactose fermenting ability.

\section{Main Sensory Attributes}

As shown in Figure 4, no significant differences were observed in firmness, sweetness, and astringency in the 3 kinds of yogurt samples. However, SYX had unique scores for sourness and butter flavor: sourness: $8.6 \pm$ 0.5 and astringent: $3.4 \pm 0.2$. Scores for butter flavor in SY fermented by Lb. pentosus without sugar (control group) were lowest $(2.6 \pm 0.2)$ in these 3 samples; this is consistent with the results of 2,3-butanedione release $(\mathrm{RC}=10.13 \%)$ in the xylose group (Table 2$)$. These data indicate that xylose addition takes a positive part in butter flavor formation (Anderson et al., 2013). As expected, the firmness, sweetness, and sourness scores of the xylose group also revealed the preferred sensory quality evaluation: firmness: $8.7 \pm 0.3$, sweetness: $6.8 \pm$ 0.4 , and butter flavor: $5.9 \pm 0.5$.

\section{Activity of ALS and ALDB in Carbohydrate Metabolism}

The production of diacetyl (2,3-butanedione) during milk fermentation was shown to depend on the strain, $\mathrm{pH}$, and temperature (Pakdeeto et al., 2003). In this study, 2,3-butanedione released in the $L b$. pentosus fermentation also had significant differences among different carbon sources (glucose, lactose, and xylose), with $\mathrm{RC}$ values from 1.74 to $7.77 \%$. Moreover, 2,3-butanedione is a metabolic end product synthesized from pyruvate anaerobically in milk fermentation. For optimal production of the potent flavor compounds ethanol, 
2,3-butanedione, and acetic acid in yogurt fermented by $L b$. pentosus, more efficient chemical conversion of carbohydrate into VOC should be developed.

Lactic acid bacteria show homolactic metabolism when growing in glucose (Papagianni, 2012). For the beneficial use of lactose and xylose in industrial milk products, we explored the carbohydrate metabolism by Lb. pentosus. As the data revealed above, milk fermented by $L b$. pentosus was efficient and had the ability to potentially produce VOC, such as ethanol, 2,3-butanedione, and acetic acid. In the metabolic pathway (Figure 5), ALS and ALDB have important roles in milk fermentation. Increasing the activities of ALS and ALDB would be effective in milk fermentation. From this point of view, the activities of ALS and ALDB were measured in vitro at different $\mathrm{pH}$ and temperatures. As illustrated in Figure 6, the preferable conditions for ALS and ALDB were $\mathrm{pH} 5.5$ and $42^{\circ} \mathrm{C}$. Under these conditions, the RC of potent flavor compounds in SYX were also determined by SPME-GC-MS (shown in Table 2); the RC of 2,3-butanedione was $10.13 \%$, which was $2.55 \%$ higher than at common culture conditions $\left(\mathrm{pH} 4.5\right.$ and $\left.37^{\circ} \mathrm{C}\right)$. The reason why the concentration of 2,3-butanedione produced was enhanced may be due to genes required for xylose utilization being derepressed under lower concentrations of glucose and also the optimal culture conditions for ALS and ALDB.

\section{CONCLUSIONS}

Xylose ( $1 \%$ addition) metabolized by $L b$. pentosus strongly affected the flavor of yogurt, with the potent VOC ethanol (3.08\%), 2,3-butanedione (7.77\%), and acetic acid (22.70\%) detected using SPME-GC-MS analysis. Meanwhile, ALS and ALDB exhibited an efficient capacity of converting carbohydrate into potent VOC (acetic acid, 2,3-butanedione, and ethanol) under optimal culture conditions $\left(42^{\circ} \mathrm{C}\right.$ and $\left.\mathrm{pH} 5.5\right)$ in this research. With the need for making health and nutrition claims and also good sensory properties, xylose metabolized by $L b$. pentosus has potential value for the milk product industry. However, further research is necessary to identify the capacity of ALS and ALDB in vitro to produce the naturalness and agreeable taste of yogurt.

\section{ACKNOWLEDGMENTS}

This work was supported by the Natural Science Foundation of China (Beijing, China; 41276121) and Zhejiang Province (China; Z3110211), the Science and Technology Department of Ningbo (China; 2012B82017, 2011C11017), and the K. C. Wong Magna Fund at Ningbo University (Ningbo, China).

\section{REFERENCES}

Abete, I., E. Goyenechea, M. A. Zulet, and J. A. Martínez. 2011. Obesity and metabolic syndrome: Potential benefit from specific nutritional components. Nutr. Metab. Cardiovasc. Dis. 21:B1-B15.

Anderson, S. E., J. Franko, J. R. Wells, E. Lukomska, and B. J. Meade. 2013. Evaluation of the hypersensitivity potential of alternative butter flavorings. Food Chem. Toxicol. 62:373-381.

Ao, X., X. Zhang, L. Shi, K. Zhao, J. Yu, L. Dong, Y. Cao, and Y. Cai. 2012. Identification of lactic acid bacteria in traditional fermented yak milk and evaluation of their application in fermented milk products. J. Dairy Sci. 95:1073-1084.

Bustos, G., A. B. Moldes, J. M. Cruz, and J. M. Domínguez. 2005. Influence of the metabolism pathway on lactic acid production from hemicellulosic trimming vine shoots hydrolyzates using Lactobacillus pentosus. Biotechnol. Prog. 21:793-798.

Chaillou, S., P. H. Pouwels, and P. W. Postma. 1999. Transport of Dxylose in Lactobacillus pentosus, Lactobacillus casei, and Lactobacillus plantarum: Evidence for a mechanism of facilitated diffusion via the phosphoenolpyruvate:mannose phosphotransferase system. J. Bacteriol. 181:4768-4773.

Chen, M. Z., L. Trinnaman, K. Bardsley, C. J. St Hilaire, and N. C. Da Costa. 2011. Volatile compounds and sensory analysis of both harvests of double-cut Yakima peppermint (Mentha piperita L.). J. Food Sci. 76:C1032-C1038.

Cheng, H. 2010. Volatile flavor compounds in yogurt: A review. Crit. Rev. Food Sci. Nutr. 50:938-950.

De Vuyst, L., and M. Vancanneyt. 2007. Biodiversity and identification of sourdough lactic acid bacteria. Food Microbiol. 24:120-127.

Delgado, F. J., J. González-Crespo, R. Cava, and R. Ramírez. 2011. Changes in the volatile profile of a raw goat milk cheese treated by hydrostatic high pressure at different stages of maturation. Int. Dairy J. 21:135-141.

Grigioni, G. M., C. A. Margaría, N. A. Pensel, G. Sánchez, and S. R. Vaudagna. 2000. Warmed-over flavour analysis in low temperature-long time processed meat by an "electronic nose". Meat Sci. $56: 221-228$.

Herve-Jimenez, L., I. Guillouard, E. Guedon, S. Boudebbouze, P. Hols, V. Monnet, E. Maguin, and F. Rul. 2009. Postgenomic analysis of streptococcus thermophilus cocultivated in milk with Lactobacillus delbrueckii ssp. bulgaricus: Involvement of nitrogen, purine, and iron metabolism. Appl. Environ. Microbiol. 75:2062-2073.

Hugenholtz, J., M. Kleerebezem, M. Starrenburg, J. Delcour, W. de Vos, and P. Hols. 2000. Lactococcus lactis as a cell factory for highlevel diacetyl production. Appl. Environ. Microbiol. 66:41124114.

Hugenholtz, J., and M. J. C. Starrenburg. 1992. Diacetyl production by different strains of Lactococcus lactis subsp. lactis var. diacetylactis and Leuconostoc spp. Appl. Microbiol. Biotechnol. $38: 17-22$.

Kühn, J., T. Considine, and H. Singh. 2006. Interactions of milk proteins and volatile flavor compounds: Implications in the development of protein foods. J. Food Sci. 71:R72-R82.

Law, J., and A. Haandrikman. 1997. Proteolytic enzymes of lactic acid bacteria. Int. Dairy J. 7:1-11.

Lee, S.-C., J. Kim, I.-J. La, S.-K. Kim, and M.-Y. Yoon. 2013. Characterization of recombinant FAD-independent catabolic acetolactate synthase from Enterococcus faecalis V583. Enzyme Microb. Technol. 52:54-59.

Majcher, M. A., and H. H. Jeleń. 2011. Key odorants of Oscypek, a traditional Polish ewe's milk cheese. J. Agric. Food Chem. 59:4932-4937.

Mamat, M., S. A. Samad, and M. A. Hannan. 2011. An electronic nose for reliable measurement and correct classification of beverages. Sensors (Basel) 11:6435-6453.

Monnet, C., V. Phalip, P. Schmitt, and C. Diviès. 1994. Comparison of $\alpha$-acetolactate synthase and $\alpha$-acetolactate decarboxylase in Lactococcus spp. and Leuconostoc spp. Biotechnol. Lett. 16:257-262.

Pakdeeto, A., N. Naranong, and S. Tanasupawat. 2003. Diacetyl of lactic acid bacteria from milk and fermented foods in Thailand. J. Gen. Appl. Microbiol. 49:301-307. 
Papagianni, M. 2012. Recent advances in engineering the central carbon metabolism of industrially important bacteria. Microb. Cell Fact. 11:50.

Peng, T., D. Pan, X. Zeng, and X. Cao. 2011. Optimization of screening and fermentation conditions of lactic acid bacteria for diacetyl production. J. Chin. Inst. Food Sci. Technol. 11:34-41.

Rabot, S., J. Rafter, G. T. Rijkers, B. Watzl, and J.-M. Antoine. 2010. Guidance for substantiating the evidence for beneficial effects of probiotics: Impact of probiotics on digestive system metabolism. J. Nutr. 140:677S-689S.

Roberts, D. D., P. Pollien, and C. Milo. 2000. Solid-phase microextraction method development for headspace analysis of volatile flavor compounds. J. Agric. Food Chem. 48:2430-2437.

Salvador, A., and S. M. Fiszman. 2004. Textural and sensory characteristics of whole and skimmed flavored set-type yogurt during long storage. J. Dairy Sci. 87:4033-4041.
Sodini, I., P. Morin, A. Olabi, and R. Jiménez-Flores. 2006. Compositional and functional properties of buttermilk: A comparison between sweet, sour, and whey buttermilk. J. Dairy Sci. 89:525-536.

Soukoulis, C., E. Aprea, F. Biasioli, L. Cappellin, E. Schuhfried, T. D. Mark, and F. Gasperi. 2010. Proton transfer reaction time-of-flight mass spectrometry monitoring of the evolution of volatile compounds during lactic acid fermentation of milk. Rapid Commun. Mass Spectrom. 24:2127-2134.

Soukoulis, C., P. Panagiotidis, R. Koureli, and C. Tzia. 2007. Industrial yogurt manufacture: Monitoring of fermentation process and improvement of final product quality. J. Dairy Sci. 90:2641-2654

Yu, H., and J. Wang. 2007. Discrimination of LongJing green-tea grade by electronic nose. Sensor. Actuat. B Chem. 122:134-140.

Zareba, D., M. Ziarno, and M. Obiedzinski. 2012. Volatile profile of non-fermented milk and milk fermented by Bifidobacterium Animalis subsp. lactis. Int. J. Food Prop. 15:1010-1021. 